\title{
Violences physiques vs violences psychologiques
}

\section{Hans Stalder}

Prof. Dr, membre de la rédaction

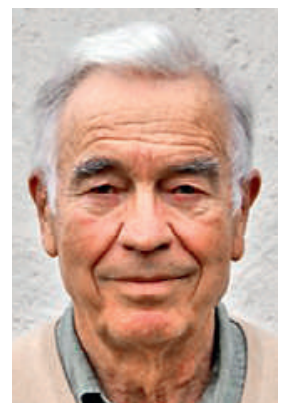

La violence a existé depuis que l'homme a été chassé du paradis. Le premier acte de violence familiale est cependant atypique, car la victime, Abel, n'est pas une femme. En effet, pratiquement toutes les statistiques des consultations traitant des problèmes de violence montrent qu'environ $3 / 4$ des victimes sont des femmes $[1,2]$. C'est probablement pour cette raison que certaines de ces consultations sont situées dans des départements de gynécologie et d'obstétrique [3], endroit pas particulièrement propice pour des hommes ayant subi des violences et désirant consulter...

Que la victimisation a toujours été principalement associée à des femmes [2] est probablement dû au fait que les femmes sont plus vulnérables aux agressions physiques, étant donné qu'elles sont en général moins fortes que les hommes. Les agressions physiques sont souvent évidentes et donc faciles à détecter. Mais les femmes sont-elles véritablement moins violentes que les hommes? On peut en douter si l'on ne considère pas uniquement les agressions physiques, mais aussi les violences psychologiques. Celles-ci ne sont pas seulement plus courantes, elles sont aussi réparties également parmi les sexes, avec quelquefois même une prédominance féminine [4]. Les violences psychologiques sont cependant beaucoup plus difficiles à déceler que les violences physiques, ce qui est regrettable, car elles peuvent causer des séquelles plus graves chez les victimes que les violences physiques [5].

La violence physique est de moins en moins supportée dans notre civilisation. Beaucoup de pays européens ont interdit la punition physique des enfants. En Suisse ce n'est pas encore le cas, mais qui oserait de nos jours donner une «bonne» claque en public à un enfant désobéissant?

Chez nous, un condamné sera enfermé, et selon la gravité du cas même en cellule d'isolement, plutôt que soumis à une punition physique, par exemple une centaine de coups de fouet comme en Arabie saoudite. Le rapport à la violence est donc fortement influencé par l'environnement culturel: ce qui était chez nous accepté autrefois est encore admis dans d'autres pays. Mais est-ce qu'un emprisonnement prolongé en cellule d'isolement laisse moins de séquelles psychologiques qu'une centaine de coups de fouet?

Ces différences de perception existent aussi pour les violences psychologiques. Prenons l'exemple du blas- phème, qui nous a occupés ces dernières semaines suite au terrible assassinat des reporters de Charlie Hebdo. Le blasphème, la parole ou le discours qui outrage la divinité, la religion ou ce qui est considéré comme respectable ou sacré (Larousse), peut être considéré soit comme non punissable (en France par exemple) ou comme une terrible violence psychologique, selon le milieu culturel où l'on vit. Chez nous sa perception a changé au cours des siècles: Thomas d'Aquin, un de nos Pères de l'Eglise, considérait encore le blasphème comme plus grave que l'homicide, et à Genève Michel Servet a été condamné au bûcher par les calvinistes pour ce qu'ils ont considéré comme un blasphème. Ce préjugé prévaut encore dans d'autres pays. Ainsi, une caricature de Mahomet qui nous fait peutêtre rire (ou pas) peut profondément blesser autrui et être considérée comme une violence psychologique extrême; le fait qu'aujourd'hui, dans notre monde globalisé, de tels dessins sont immédiatement communiqués partout et atteignent des publics d'autres cultures, peut avoir les conséquences inattendues et graves qu'on connaît.

La violence psychologique n'est donc pas seulement plus difficile et plus compliquée à investiguer parce qu'elle ne laisse pas de séquelles immédiatement visibles, mais aussi parce qu'il faut considérer le vécu culturel de la victime: une expression vocale, écrite ou dessinée jugée anodine peut être ressentie par l'autre comme violence extrême.

Tout le monde sera certainement d'accord que la violence concerne aussi la médecine [6], mais il ne faut pas se restreindre aux séquelles de la violence physique causées par les hommes, mais aussi considérer les violences psychologiques, où femmes et hommes sont égaux.

\section{Références}

1 Criminalité et exécution des peines. Victimes. Office fédéral de la statistique. Neuchâtel; 2015.

2 WHO multi-country study on women's health and domestic violence against women. Initial results on prevalence, health outcomes and women's responses. Genève: WHO ;2005.

3 Tschudin S. Prävention und Bekämpfung von häuslicher Gewalt. Bull Méd Suisses. 2015;96(7):243-4.

4 McHugh MC, Frieze IH. Intimate partner violence: New directions. Ann N Y Acad Sci. 2006;1087:121-41.

5 Coker AL, Smith PH, Bethea L, King MR, McKeown RE. Physical health consequences of physical and psychological intimate partner violence. Arch Fam Med. 2000;9:451-7.

6 Romann C. La violence domestique: un sujet qui concerne aussi la médecine. Bull Méd Suisses. 2015;96(7):213. 